\title{
"Factorisatio numerorum" in arithmetical semigroups
}

\author{
by \\ ARnOld KnOpFmaCher (Johannesburg), \\ JOHN KNOPFMACHER (Johannesburg) \\ and RichaRD WARLIMONT (Regensburg)
}

Introduction. The problems of "factorisatio numerorum", which go back more than 65 years, are concerned principally with (i) the total number $f(n)$ of factorizations of a natural number $n>1$ into products of natural numbers larger than 1 , where the order of the factors is not counted, and (ii) the corresponding total number $F(n)$ of factorizations when the order of the factors is counted. For example, $f(12)=4$ while $F(12)=8$. Some results and further references on such problems may be found in [1], [2], [5], [6] and [7] in particular.

The object of this paper is to consider similar problems and results, with emphasis on the average numbers of factorizations of each kind, within the partly analogous but also quite distinct context of additive arithmetical semigroups. Such semigroups (to be defined below) are treated in the monographs [3], [4] within an abstract setting designed to conveniently cover (under minimal assumptions) concrete cases like (i) the multiplicative semigroup $G_{q}$ of all monic polynomials in one indeterminate over a finite field $\mathbb{F}_{q}$, (ii) semigroups of ideals in principal orders within algebraic function fields over $\mathbb{F}_{q}$, (iii) semigroups formed under direct sum by the isomorphism classes of certain kinds of finite modules or algebras over such principal orders.

Although the first main result below will be derived within a still more general framework, we shall formulate and state our second main conclusion within a context (see [4]) which conveniently covers the preceding concrete cases:

An (additive) arithmetical semigroup will be understood to be a free commutative semigroup $G$ with identity element 1 , generated by a (countable) set $P$ of "prime" elements, which admits an integer-valued "degree"

1991 Mathematics Subject Classification: 11N37, 11N80. 
mapping $\partial$ such that

(i) $\partial(1)=0, \partial(p)>0$ for all $p \in P$,

(ii) $\partial(a b)=\partial(a)+\partial(b)$ for all $a, b \in G$,

(iii) the total number $G^{\#}(n)$ of elements of degree $n$ in $G$ is finite for all $n \in \mathbb{N}$.

All the concrete cases referred to above provide examples of an arithmetical semigroup $G$ which satisfies

Axiom A\#. There exist constants $A>0, q>1$, and $\nu$ with $0 \leq \nu<1$ such that

$$
G^{\#}(n)=A q^{n}+O\left(q^{\nu n}\right) \quad \text { as } n \rightarrow \infty .
$$

In dealing with an arithmetical semigroup $G$, it is frequently helpful to consider the "zeta" (or "generating") function

$$
Z(y)=Z_{G}(y)=\sum_{n=0}^{\infty} G^{\#}(n) y^{n} .
$$

Under Axiom $\mathrm{A}^{\#}, Z(y)$ is holomorphic for $|y|<q^{-1}$, and extends to a meromorphic function in the disc $|y|<q^{-\nu}$, having exactly one pole (of order 1) at $y=q^{-1}$. In fact,

$$
Z(y)=\frac{A}{1-q y}+h_{1}(y)
$$

where $h_{1}(y)$ is holomorphic for $|y|<q^{-\nu}$.

Now consider the functions $f(a), F(a)$ of $a \neq 1$ in $G$ which are directly analogous to the factorization-counting functions $f(n), F(n)$ earlier, and let $f(1)=F(1)=1$. Define

$$
\bar{f}(n)=\sum_{\partial(a)=n} f(a), \quad \bar{F}(n)=\sum_{\partial(a)=n} F(a) .
$$

THEOREM 1. Let $G \neq\{1\}$ be an arithmetical semigroup having a zeta function $Z(y)$ which is holomorphic in some disc $|y|<r$ and takes values $>2$ for some real values of $y<r$. Then

$$
\bar{F}(n)=\frac{y_{0}^{-n-1}}{Z^{\prime}\left(y_{0}\right)}+O\left(t_{0}^{-n}\right) \quad \text { as } n \rightarrow \infty,
$$

where $y_{0} \in(0, r)$ is the unique real solution of $Z(y)-2=0$, and $y_{0}<t_{0}<r$.

In particular, under Axiom $\mathrm{A}^{\#}$ the average number of ordered factorizations of an element of degree $n$ in $G$ has the form

$$
\frac{\bar{F}(n)}{G^{\#}(n)}=\frac{q^{-n} y_{0}^{-n-1}}{A Z^{\prime}\left(y_{0}\right)}+O\left(\left(q t_{0}\right)^{-n}\right)
$$

where $y_{0}<t_{0}<q^{-1}$. 
This theorem provides an analogue to one of Kalmár [2] for natural numbers, in which $Z(y)$ is replaced by the Riemann zeta function. It is applicable not only to the explicit examples outlined earlier, but also to many other quite different concrete cases, as listed in [3], Chapter 8, say. Theorem 1 also provides an asymptotic generalization of the conclusion valid for the special semigroup $G=G_{q}$ above, for which $G^{\#}(n)=q^{n}, Z(y)=$ $(1-q y)^{-1}, y_{0}=(2 q)^{-1}$, and $\bar{F}(n)=2^{n-1} q^{n}$ exactly when $n>0$. (Here the exact equation for $\bar{F}(n)$ may be deduced either with the aid of that for $Z(y)$ or by simple combinatorial reasoning: Factorizations of polynomials of degree $n$ into factors of degrees $n_{1}, \ldots, n_{m}$ respectively, where $n_{1}+\ldots+$ $n_{m}=n$, can be formed in $q^{n_{1}} \ldots q^{n_{m}}=q^{n}$ ways. Since we have $2^{n-1}$ such compositions of $n$, the result follows.)

As happens for natural numbers according to Oppenheim [5] and Szekeres and Turán [7], the result for unordered factorizations under Axiom $\mathrm{A}^{\#}$ is more involved than that for the ordered case:

TheOREM 2. Under Axiom $\mathrm{A}^{\#}$, the average number of unordered factorizations of an element of degree $n$ in $G$ has the form

$$
\frac{\bar{f}(n)}{G^{\#}(n)}=C_{G} n^{-3 / 4} \exp (2 \sqrt{A n})\left(1+O\left(n^{-1 / 2}\right)\right) \quad \text { as } n \rightarrow \infty,
$$

where

$$
C_{G}=\frac{A^{-3 / 4} e^{A / 2}}{2 \sqrt{\pi}} \exp \left(\sum_{m=0}^{\infty}\left(G^{\#}(m)-A q^{m}\right) q^{-m}+\sum_{k=2}^{\infty} \frac{Z\left(q^{-k}\right)-1}{k}\right) .
$$

In the unordered case covered by this theorem, the conclusion remains nontrivial even for the very special semigroup $G_{q}$ earlier: For $G_{q}$, the average has the form

$$
\frac{\bar{f}(n)}{q^{n}}=\frac{1}{2} \sqrt{\frac{e}{\pi}} \exp \left(\sum_{k=1}^{\infty} \frac{1}{(k+1)\left(q^{k}-1\right)}\right) n^{-3 / 4} \exp (2 \sqrt{n})\left(1+O\left(n^{-1 / 2}\right)\right) .
$$

At the expense of more involved computational analysis (which we may return to elsewhere), it seems possible to replace the asymptotic formula of Theorem 2 by an asymptotic series of arbitrary length (cf. [7] for natural numbers).

1. Ordered factorizations. For any (additive) arithmetical semigroup $G$, the total number $F_{k}(a)$ of ordered factorizations of $a \neq 1$ into exactly $k \geq 1$ factors in $G$ has the formal generating function

$$
\sum_{1 \neq a \in G} F_{k}(a) y^{\partial(a)}=\left(\sum_{1 \neq b \in G} y^{\partial(b)}\right)^{k}=(Z(y)-1)^{k} .
$$


Since $F(a)=\sum_{k=1}^{\infty} F_{k}(a)$, this formally implies that

$$
\sum_{n=0}^{\infty} \bar{F}(n) y^{n}=\sum_{k=0}^{\infty}(Z(y)-1)^{k}=(2-Z(y))^{-1} .
$$

Under Axiom $\mathrm{A}^{\#}$, this relation will be analytically valid for $|y|<y_{0}$, where $y_{0} \in\left(0, q^{-1}\right)$ is the real solution of $Z(y)-2=0$ :

LeMma 1.1. For any arithmetical semigroup $G \neq\{1\}$ having a zeta function $Z(y)$ which is holomorphic in some disc $|y|<r$ and takes values $>2$ for some real $y<r$, the equation $Z(y)-2=0$ has a unique and simple root $y_{0} \in(0, r)$, but no other complex root $y$ with $|y| \leq y_{0}$.

Proof. Since $Z(y)$ is strictly increasing and passes the value 2 on $(0, r)$, there must be a unique $y_{0} \in(0, r)$ with $Z\left(y_{0}\right)=2$. Since $Z^{\prime}\left(y_{0}\right)>0, y_{0}$ is a simple zero of $Z(y)-2=0$. Next, if there was a complex root $y_{1}$ of $Z(y)-2=0$ with $\left|y_{1}\right|<y_{0}$, we would have $2=Z\left(y_{1}\right)$ and thus

$$
2=\left|Z\left(y_{1}\right)\right| \leq \sum_{n=0}^{\infty} G^{\#}(n)\left|y_{1}\right|^{n}<\sum_{n=0}^{\infty} G^{\#}(n) y_{0}^{n}=2
$$

which is impossible. Further, if $y_{2}$ was a complex root with $y_{2}=y_{0} e^{i \theta}, \theta \notin$ $2 \pi \mathbb{Z}$, we would have

$$
2=Z\left(y_{2}\right)=\operatorname{Re}\left(Z\left(y_{2}\right)\right)=\sum_{n=0}^{\infty} G^{\#}(n) y_{0}^{n} \cos n \theta<\sum_{n=0}^{\infty} G^{\#}(n) y_{0}^{n}=2,
$$

which is also impossible.

Under the rather light assumptions on $G$ in Lemma 1.1, the function

$$
(2-Z(y))^{-1}=\sum_{n=0}^{\infty} \bar{F}(n) y^{n}
$$

satisfies the hypothesis on $g(y)$ in Lemma 1.2 below, and consequently, Theorem 1 follows directly from that lemma.

LEMMA 1.2. Let $h(y)$ be holomorphic for $|y|<r$, and let $w_{0}=h\left(y_{0}\right)$ for some complex $y_{0}$ with $0<\left|y_{0}\right|<r$. Suppose that $h^{\prime}\left(y_{0}\right) \neq 0$ and $h(y) \neq w_{0}$ for $|y| \leq\left|y_{0}\right|, y \neq y_{0}$. Then, if

$$
g(y):=\left(w_{0}-h(y)\right)^{-1}=\sum_{n=0}^{\infty} a(n) y^{n} \quad \text { for }|y|<\left|y_{0}\right|,
$$

there exists $t_{0}$ with $\left|y_{0}\right|<t_{0}<r$ such that

$$
a(n)=\frac{y_{0}^{-n-1}}{h^{\prime}\left(y_{0}\right)}+O\left(t_{0}^{-n}\right) \quad \text { as } n \rightarrow \infty .
$$


Proof. Since $h^{\prime}\left(y_{0}\right) \neq 0, y_{0}$ is a simple zero of $h(y)-w_{0}=0$. Thus $g(y)$ is holomorphic for $|y| \leq\left|y_{0}\right|$, apart from a simple pole at $y=y_{0}$. In fact $g(y)$ remains otherwise holomorphic for $|y|<r_{0}$ where $\left|y_{0}\right|<r_{0}<r$, and such an $r_{0}$ exists because: (i) every $y \neq y_{0}$ with $|y|=\left|y_{0}\right|$ is the centre of some disc of holomorphy for $g(y)$, and (ii) $y_{0}$ is the centre of some disc in which $h(y)-w_{0}=0$ has no other zero, i.e. $g(y)$ is holomorphic in that disc apart from the pole at $y=y_{0}$; hence $r_{0}$ exists by compactness of the circle $|y|=\left|y_{0}\right|$.

Now choose any $t_{0}$ with $\left|y_{0}\right|<t_{0}<r_{0}$, and put

$$
J(n)=\frac{1}{2 \pi i} \int_{|y|=t_{0}} \frac{1}{w_{0}-h(y)} \frac{d y}{y^{n+1}} .
$$

On the one hand, $J(n)=O\left(t_{0}^{-n}\right)$, and, on the other, Cauchy's residue theorem yields

$$
J(n)=a(n)-\frac{y_{0}^{-n-1}}{h^{\prime}\left(y_{0}\right)}
$$

This proves the lemma.

Remarks. (i) At the expense of a slightly weaker error estimate, Theorem 1 could also be derived with the aid of Lemma 7.3 of [4], page 60 .

(ii) In considering concrete examples of semigroups satisfying Axiom $\mathrm{A}^{\#}$, as indicated in the Introduction, cases occur in which the fixed constant $q$ is an arbitrarily large prime-power. There is therefore some interest in considering the behaviour of $y_{0}$ and of the average $F(n) / G^{\#}(n)$ as $q \rightarrow \infty$. One then obtains the approximate values

$$
y_{0} \sim \frac{1}{(A+1) q}, \quad Z_{G}^{\prime}\left(y_{0}\right) \sim \frac{q}{A}(A+1)^{2}, \quad \frac{\bar{F}(n)}{G^{\#}(n)} \sim(A+1)^{n-1},
$$

which may be compared with the earlier-mentioned conclusions for the special semigroup $G_{q}$ (for which $A=1$ ).

2. Unordered factorizations. For any arithmetical semigroup $G$ we may formally write

$$
\begin{aligned}
f^{\#}(y): & =\sum_{n=0}^{\infty} \bar{f}(n) y^{n}=\sum_{a \in G} f(a) y^{\partial(a)}=\prod_{1 \neq b \in G}\left(1+y^{\partial(b)}+y^{2 \partial(b)}+\ldots\right) \\
& =\prod_{1 \neq b \in G}\left(1-y^{\partial(b)}\right)^{-1}=\prod_{m=1}^{\infty}\left(1-y^{m}\right)^{-G^{\#}(m)} .
\end{aligned}
$$

If $Z(y)$ is holomorphic in a disc $|y|<r$, the resulting Cauchy inequality for $G^{\#}(m)$ implies that the last product converges uniformly on every disc 
$|y| \leq t<r$. Thus $f^{\#}(y)$ is holomorphic, and

$$
f^{\#}(y)=\exp \left(\sum_{m=1}^{\infty} G^{\#}(m) \log \left(1-y^{m}\right)^{-1}\right)=\exp \left(\sum_{k=1}^{\infty} \frac{Z\left(y^{k}\right)-1}{k}\right),
$$

for $|y|<\min \{r, 1\}$, on expanding $\log \left(1-y^{m}\right)=-\sum_{k=1}^{\infty} y^{m k} / k$ and interchanging orders of summation. Further

$$
f^{\#}(y)=\exp \left(Z(y)-1+h_{2}(y)\right),
$$

where $h_{2}(y)$ is holomorphic for $|y|<\sqrt{r}$, if $Z(y)$ is holomorphic for $|y|<$ $r<1,0<r$ (so that $G^{\#}(m) \ll r_{1}^{m}$ for $0<r_{1}<r$ ): To see this, let $|y|<r_{2}$ for $0<r_{2}<\sqrt{r_{1}}<\sqrt{r}$. Then

$$
\begin{aligned}
h_{2}(|y|) & =\sum_{k=2}^{\infty} \frac{1}{k} \sum_{m=1}^{\infty} G^{\#}(m)|y|^{k m} \ll \sum_{k=2}^{\infty} \sum_{m=1}^{\infty}\left(r_{1}^{-1} r_{2}^{k}\right)^{m} \\
& \leq \sum_{k=2}^{\infty} r_{1}^{-1} r_{2}^{k} \sum_{m=0}^{\infty}\left(r_{1}^{-1} r_{2}^{2}\right)^{m}<\infty
\end{aligned}
$$

since $r_{2}<1, r_{1}^{-1} r_{2}^{2}<1$.

Now suppose that $G$ satisfies Axiom A $\mathrm{A}^{\#}$, so that we can take $r=q^{-1}$ above. Then, as stated earlier,

$$
Z(y)=\frac{A}{1-q y}+h_{1}(y)
$$

where $h_{1}(y)$ is holomorphic for $|y|<q^{-\nu}$, and we obtain

$$
f^{\#}(y)=\exp \left(\frac{A}{1-q y}\right) g(y),
$$

where $g(y)=\exp \left(h_{1}(y)-1+h_{2}(y)\right)$ is holomorphic for $|y|<\min \left(q^{-\nu}, q^{-1 / 2}\right)$. It follows that $f^{*}(w)=E(w) g^{*}(w)$, where $f^{*}(w)=f^{\#}(w / q), E(w)=$ $\exp (A /(1-w)), g^{*}(w)=g(w / q)$, and we have expansions

$$
\begin{aligned}
E(w) & =\sum_{n=0}^{\infty} \varepsilon(n) w^{n} \quad \text { for }|w|<1, \\
g^{*}(w) & =\sum_{n=0}^{\infty} \delta(n) w^{n} \quad \text { for }|w|<r_{1}:=\min \left(q^{1-\nu}, q^{1 / 2}\right),
\end{aligned}
$$

and $f^{*}(w)=\sum_{n=0}^{\infty} \beta(n) w^{n}$ with $\beta(n)=\bar{f}(n) q^{-n}$.

In order to prove Theorem 2, we shall estimate $\beta(n)$ by the Cauchy integral formula

$$
\beta(n)=\frac{1}{2 \pi i} \int_{L} E(w) g^{*}(w) \frac{d w}{w^{n+1}}
$$


where $L$ is the contour

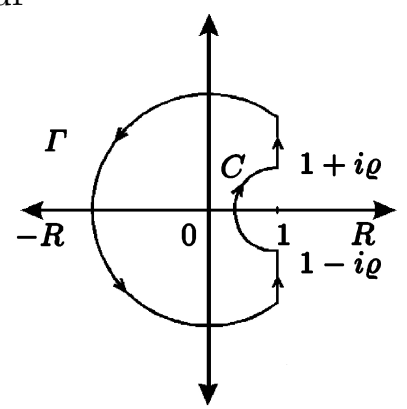

in which $\Gamma$ consists of the indicated vertical segments plus portion of the circle $|w|=R, R=\frac{1}{2}\left(r_{1}+1\right)$, and $C$ is the indicated semicircle of radius $\varrho=\sqrt{A / n}$ about $w=1$, with $\varrho<1$ (for $n>n_{0}$, say). Thus for $n>n_{0}$

$$
\beta(n)=\frac{1}{2 \pi i}\left(\int_{C}+\int_{\Gamma}\right)=\frac{1}{2 \pi i} \int_{C}+O(1) .
$$

On $C$ we have

$$
g^{*}(w)=g^{*}(1)+g^{* \prime}(1)(w-1)+O\left(\varrho^{2}\right),
$$

where $w=1-\varrho e^{-i \theta}(-\pi / 2 \leq \theta \leq \pi / 2)$. So

$$
\frac{1}{2 \pi i} \int_{C}=g^{*}(1) J_{1}(n)+g^{* \prime}(1) J_{2}(n)+J_{3}(n)
$$

where

$$
\begin{aligned}
& J_{1}(n)=\frac{\varrho}{2 \pi} \int_{-\pi / 2}^{\pi / 2} \exp \left(\frac{A}{\varrho} e^{i \theta}\right) \frac{e^{-i \theta}}{\left(1-\varrho e^{-i \theta}\right)^{n+1}} d \theta, \\
& J_{2}(n)=\frac{-\varrho^{2}}{2 \pi} \int_{-\pi / 2}^{\pi / 2} \exp \left(\frac{A}{\varrho} e^{i \theta}\right) \frac{e^{-2 i \theta}}{\left(1-\varrho e^{-i \theta}\right)^{n+1}} d \theta,
\end{aligned}
$$

and the similar integral

$$
\begin{aligned}
J_{3}(n) & \ll \varrho^{3} \exp \left(\frac{A}{\varrho}\right) \frac{1}{(1-\varrho)^{n+1}} \ll \varrho^{3} \exp \left(\frac{A}{\varrho}+n \varrho\right)\left(\frac{e^{-\varrho}}{1-\varrho}\right)^{n} \\
& \ll \varrho^{3} \exp \left(\frac{2 A}{\varrho}\right),
\end{aligned}
$$

since

$$
\varrho=\sqrt{A / n}, \quad n \varrho=A / \varrho, \quad(1-\varrho)^{-1}=1+O(\varrho),
$$

and

$$
\left(\frac{e^{-\varrho}}{1-\varrho}\right)^{n}=\exp \left(n\left(-\varrho+\log \frac{1}{1-\varrho}\right)\right) \ll \exp \left(n \varrho^{2}\right)=e^{A}
$$


Next, if $H(\theta)=\frac{\exp \left(-\varrho e^{-i \theta}\right)}{1-\varrho e^{-i \theta}}$, then

$$
\begin{aligned}
J_{2}(n) & =\frac{-\varrho^{2}}{2 \pi} \int_{-\pi / 2}^{\pi / 2} \exp \left(\frac{A}{\varrho} e^{i \theta}+n \varrho e^{-i \theta}\right)(H(\theta))^{n} \frac{e^{-2 i \theta}}{1-\varrho e^{-i \theta}} d \theta \\
& =\frac{-\varrho^{2}}{2 \pi} \exp \left(\frac{2 A}{\varrho}\right) \int_{-\pi / 2}^{\pi / 2} \exp \left(-4 \frac{A}{\varrho} \sin ^{2} \frac{\theta}{2}\right)(H(\theta))^{n} \frac{e^{-2 i \theta}}{1-\varrho e^{-i \theta}} d \theta,
\end{aligned}
$$

since

$$
\frac{A}{\varrho} e^{i \theta}+n \varrho e^{-i \theta}=\frac{A}{\varrho}\left(e^{i \theta}+e^{-i \theta}\right)=\frac{2 A}{\varrho} \cos \theta=\frac{2 A}{\varrho}\left(1-2 \sin ^{2} \frac{\theta}{2}\right) .
$$

Also

$$
\begin{aligned}
H(\theta) & =\exp \left(-\varrho e^{-i \theta}+\log \left(\frac{1}{1-\varrho e^{-i \theta}}\right)\right) \\
& =\exp \left(\frac{1}{2}\left(\varrho e^{-i \theta}\right)^{2}+\sum_{k=3}^{\infty} \frac{1}{k}\left(\varrho e^{-i \theta}\right)^{k}\right)=\exp \left(\frac{A e^{-2 i \theta}}{2 n}+O\left(\varrho^{3}\right)\right),
\end{aligned}
$$

and so

$$
\begin{aligned}
(H(\theta))^{n} \frac{e^{-2 i \theta}}{1-\varrho e^{-i \theta}} & =\exp \left(\frac{A}{2} e^{-2 i \theta}+O(\varrho)\right)(1+O(\varrho)) e^{-2 i \theta} \\
& =\exp \left(\frac{A}{2}\left(1-2 i \theta+O\left(\theta^{2}\right)\right)+O(\varrho)\right)(1+O(\varrho)) e^{-2 i \theta} \\
& =\exp (A / 2) \exp (-i(A+2) \theta)\left(1+O\left(\theta^{2}+\varrho\right)\right) .
\end{aligned}
$$

Thus

$$
J_{2}(n) \ll \varrho^{2} \exp \left(\frac{2 A}{\varrho}\right) \int_{-\infty}^{\infty} \exp \left(-4 \frac{A}{\varrho} \frac{\theta^{2}}{\pi^{2}}\right) d \theta,
$$

since $\sin ^{2} \frac{\theta}{2} \geq\left(\frac{2}{\pi} \frac{\theta}{2}\right)^{2}$. Hence

$$
J_{2}(n) \ll \varrho^{2} \exp \left(2 \frac{A}{\varrho}\right) \sqrt{\varrho},
$$

if one substitutes $\theta=\phi \sqrt{\varrho}$ in the integral, say.

Lastly, an argument similar to that for $J_{2}(n)$ shows that

$$
J_{1}(n)=\frac{\varrho}{2 \pi} \exp \left(2 \frac{A}{\varrho}\right) \int_{-\pi / 2}^{\pi / 2} \exp \left(-4 \frac{A}{\varrho} \sin ^{2} \frac{\theta}{2}\right) K(\theta) d \theta
$$


where

$$
\begin{aligned}
K(\theta) & =\exp \left(\frac{A}{2} e^{-2 i \theta}+O(\varrho)\right) \frac{e^{-i \theta}}{1-\varrho e^{-i \theta}} \\
& =\exp \left(\frac{A}{2}\right)(1+O(|\theta|)+O(\varrho)) .
\end{aligned}
$$

Hence

$$
J_{1}(n)=\frac{\varrho}{2 \pi} \exp \left(2 \frac{A}{\varrho}+\frac{A}{2}\right)\left(\int_{-\pi / 2}^{\pi / 2} \exp \left(-4 \frac{A}{\varrho} \sin ^{2} \theta\right) d \theta+O(\varrho)\right),
$$

since

$$
\int_{-\pi / 2}^{\pi / 2} \exp \left(-4 \frac{A}{\varrho} \sin ^{2} \frac{\theta}{2}\right)(O(|\theta|)+O(\varrho)) d \theta \ll \varrho+\varrho^{3 / 2}=O(\varrho)
$$

similarly to the discussion of the second integral for $J_{2}(n)$ above. Also

$$
\int_{-\pi / 2}^{\pi / 2} \exp \left(-4 \frac{A}{\varrho} \sin ^{2} \frac{\theta}{2}\right) d \theta=\sqrt{\frac{\varrho \pi}{A}}(1+O(\varrho))
$$

for the reasons below, and after this the proof of Theorem 2 is complete.

For the final integral, note that

$$
\exp \left(-4 \frac{A}{\varrho} \sin ^{2} \frac{\theta}{2}\right)=\left(-\frac{A}{\varrho} \theta^{2}\right) \exp \left(-4 \frac{A}{\varrho}\left(\sin ^{2} \frac{\theta}{2}-\frac{\theta^{2}}{4}\right)\right)
$$

and $\sin ^{2} \frac{\theta}{2}-\frac{\theta^{2}}{4}=O\left(\theta^{4}\right)$. Therefore

$$
\exp \left(-4 \frac{A}{\varrho}\left(\sin ^{2} \frac{\theta}{2}-\frac{\theta^{2}}{4}\right)\right)=1+O\left(\frac{\theta^{4}}{\varrho}\right) \quad \text { for }|\theta| \leq \varrho^{1 / 4},
$$

and

$$
\begin{aligned}
\int_{-\pi / 2}^{\pi / 2} & \exp \left(-4 \frac{A}{\varrho} \sin ^{2} \frac{\theta}{2}\right) d \theta \\
= & \int_{-\varrho^{1 / 4}}^{\varrho^{1 / 4}} \exp \left(-\frac{A}{\varrho} \theta^{2}\right)\left(1+O\left(\frac{\theta^{4}}{\varrho}\right)\right) d \theta+O\left(\int_{\varrho^{1 / 4}}^{\infty} \exp \left(-\frac{A}{\varrho} \frac{\theta^{2}}{\pi^{2}}\right) d \theta\right) \\
= & \int_{-\infty}^{\infty} \exp \left(-\frac{A}{\varrho} \theta^{2}\right) d \theta+O\left(\frac{1}{\varrho} \int_{-\infty}^{\infty} \exp \left(-\frac{A}{\varrho} \theta^{2}\right) \theta^{4} d \theta\right. \\
& \left.+\int_{\varrho^{1 / 4}}^{\infty} \exp \left(-\frac{A}{\varrho} \frac{\theta^{2}}{\pi^{2}}\right) d \theta\right)
\end{aligned}
$$


On substituting $\theta=\phi \sqrt{\varrho / A}$, this becomes

$$
\sqrt{\frac{\varrho \pi}{A}}+O\left(\frac{1}{\varrho} \varrho^{5 / 2}+\sqrt{\varrho} \int_{\sqrt{A} \varrho^{-1 / 4}}^{\infty} \exp \left(-\frac{\phi^{2}}{\pi^{2}}\right) d \phi\right)=\sqrt{\frac{\varrho \pi}{A}}(1+O(\varrho)) .
$$

\section{References}

[1] E. R. Canfield, P. Erdős and C. Pomerance, On a problem of Oppenheim concerning "factorisatio numerorum", J. Number Theory 17 (1983), 1-28.

[2] L. Kalmár, Über die mittlere Anzahl der Produktdarstellungen der Zahlen, Acta Litt. Sci. Szeged 5 (1931), 95-107.

[3] J. Knopfmacher, Abstract Analytic Number Theory, North-Holland/Elsevier, 1975; Dover, 1990.

[4] _- Analytic Arithmetic of Algebraic Function Fields, Dekker, 1979.

[5] A. Oppenheim, On an arithmetic function, J. London Math. Soc. 1 (1926), 205-211 and 2 (1927), 123-130.

[6] G. J. Rieger, Über die Anzahl der Produktzerlegungen ganzer Zahlen, Math. Z. 76 (1961), 226-234.

[7] G. Szekeres and P. Turán, Über das zweite Hauptproblem der "Factorisatio Numerorum", Acta Litt. Sci. Szeged 6 (1933), 143-154.

A. Knopfmacher

DEPT. OF COMP. \& APPLIED MATH.

UNIVERSITY OF THE WITWATERSRAND

JOHANNESBURG

P.O. WITS 2050

SOUTH AFRICA

R. Warlimont

FACHBEREICH MATHEMATIK

UNIVERSITÄT REGENSBURG

POSTFACH 397

D-8400 REGENSBURG

GERMANY
J. Knopfmacher

DEPT. OF MATHEMATICS UNIVERSITY OF THE WITWATERSRAND JOHANNESBURG P.O. WITS 2050 SOUTH AFRICA 